\title{
Participación de la Red de Medicina de Alta Especialidad en el logro de la equidad en salud
}

\author{
Michelle Kawa-Lazard ${ }^{1}$ y Simón KaWa-Karasik ${ }^{2 *},+$ \\ 1'Investigadora, Centro de Investigación para la Paz México (CIPMEX); ${ }^{2}$ Director General de Coordinación de los Institutos Nacionales de Salud, \\ Comisión Coordinadora de los Institutos Nacionales de Salud y Hospitales de Alta Especialidad, Ciudad de México, México
}

\section{RESUMEN}

Equidad en salud significa que, independientemente de su posición social, género, identidad sexual, ubicación geográfica o cualquier otra circunstancia, las personas pueden alcanzar su máximo potencial de salud. Por lo tanto, debemos reconocer que los determinantes sociales tales como las circunstancias en que las personas nacen, crecen, viven, trabajan y envejecen, incluido el sistema de salud en sí mismo, son elementos esenciales que se incorporarán al abordar el tema de equidad en salud. En este ensayo presentamos algunos aspectos relacionados con el acceso a los servicios de salud, y en particular a la medicina altamente especializada, así como la participación de las instituciones públicas en nuestro país desde una perspectiva de equidad y justicia social. La Secretaría de Salud, a través de la Comisión Coordinadora de los Institutos Nacionales de Salud y Hospitales de Alta Especialidad (CCINSHAE), ha integrado la Red de Medicina de Alta Especialidad, compuesta por los los Institutos Nacionales de Salud (INSALUD), los Hospitales Federales de Referencia (HFR) y los Hospitales Regionales de Alta Especialidad (HRAE). Esta red coordina sistemáticamente acciones enfocadas a promover el acceso efectivo a servicios médicos especializados en varias regiones del país con calidad y seguridad, basándose en la mejor evidencia científica posible, con el objetivo de dar atención priorizada a poblaciones de diferentes estratos socioeconómicos, y haciendo hincapié en la atención a las poblaciones vulnerables, lo que ayuda a reducir las brechas que aún existen en el acceso a los servicios de atención médica. Todo ello genera un gran impacto en la salud y agrega valor al Sistema Nacional de Salud. Sin embargo, teniendo en cuenta una concepción que lo abarca todo, está claro que el proceso salud-enfermedad está influenciado por múltiples factores, en los que los aspectos sociales tienen un peso sustancial. Por lo tanto, es necesario reunir información útil para articular nuevos enfoques y seleccionar diferentes intervenciones según cada situación para poder dar respuestas que ayuden a quienes más lo necesitan. La equidad sanitaria no debe centrarse simplemente en el acceso a los servicios de salud ni debe verse como un asunto aislado, sino que debe abordarse desde la perspectiva más amplia de imparcialidad y justicia, incluyendo aspectos económicos tales como la distribución de recursos, derechos humanos y libertad como principales criterios a ser incorporados. La equidad en la salud debe entenderse desde una perspectiva multidimensional y la comprensión de la justicia social.

Palabras clave: Equidad en salud. Medicina de alta especialidad. Justicia social.

\author{
Correspondencia: \\ *Simón Kawa-Karasik \\ Comisión Coordinadora de los Institutos Nacionales de Salud \\ y Hospitales de Alta Especialidad \\ Periférico Sur, 4118, piso 1 \\ Col. Jardines del Pedregal, Del. Álvaro Obregón \\ C.P. 01900, Ciudad de México, México \\ E mail: simon.kawa.ccinshae@gmail.com
}

+Artículo por invitación

Date of reception: 04-09-2018

Date of acceptance: 12-09-2018

Doi: 10.24875/HMCM.18000151 


\section{ABSTRACT}

Equity in Health means that regardless of social position, gender, sexual identity, geographical location or any other circumstance, people can reach their maximum health potential. Therefore, we must recognize that social determinants such as the circumstances in which people are born, grow, live, work and age, including the health system itself, are essential elements to be incorporated when addressing the issue of equity in health. In this essay, we present some aspects related to accessing to health services and in particular, to highly specialized medicine, as well as the participation of public institutions in our country from a perspective of equity and social justice. The Ministry of Health of Mexico, through the Coordinating Commission of the National Institutes of Health and High-Specialty Hospitals (CCINSHAE), has integrated a High-Specialty Medicine Network, comprised of the National Institutes of Health (INSALUD), the Federal Hospitals of Reference (HFR) and the High-Specialty Regional Hospitals (HRAE). The Network systematically and coordinatedly carries out actions focused on promoting effective access to specialized medical services in several regions in the country, with quality and safety, founded on the best possible scientific evidence. The objective is giving prioritized care to populations of different socioeconomic strata, emphasizing care to vulnerable populations, thereby helping to reduce gaps that still exist in access to health care services, generating a great health impact and adding value to the National Health System. However, considering an all-encompassing conception, clearly the healthdisease process is influenced by multiple factors, where social aspects have a substantial weight. Therefore, it is necessary to gather information that will be useful to articulate new approaches, select different interventions depending on each situation to provide answers to help those who need it the most. Health equity should not focus merely on access to health services nor should it be seen as an isolated matter; it must be addressed from a broader perspective of impartiality and justice. These require the inclusion of economic aspects such as resource distribution as well as human rights and freedom as the main criteria to be incorporated. Equity in health must be understood from a multidimensional perspective and comprehension of social justice. (HOSP MED CLIN MANAG. 2018;11:137-42)

Corresponding author: Simón Kawa-Karasik, simon.kawa.ccinshae@gmail.com

Key words: Equity in health. Highly specialized medicine. Social justice.

\section{INTRODUCCIÓN}

Fomentar la salud y el desarrollo social de sus pueblos es un compromiso esencial de los gobiernos, lo cual es compartido por amplios sectores de la sociedad. Según la Organización Mundial de la Salud (OMS), el derecho a la salud no debe entenderse únicamente como el derecho a estar sano, sino que significa que los gobiernos deben crear las condiciones que permitan a todas las personas vivir lo más saludablemente posible. Esas condiciones incluyen las disponibilidades garantizadas de servicios de salud, condiciones de trabajo saludables y seguras, vivienda adecuada y alimentos nutritivos sin distinción de raza, religión, género, ideología política o condición económica o social’ .

Teniendo en cuenta que el goce del grado máximo de salud que se pueda lograr es uno de los derechos fundamentales de todo ser humano, los propósitos primordiales para alcanzar la equidad en salud son:

- Acceso a la atención médica de calidad y a los medicamentos esenciales, especialmente para aquellas personas que se encuentran en situación de vulnerabilidad.

- Acceso a alimentación y agua adecuadas.

- Mejora de las condiciones de vida y del medio ambiente.

- Supresión de la marginación y exclusión de personas por cualquier motivo.

- Reducción de la pobreza.

Desde esta perspectiva, y ante una sociedad cada vez más compleja en donde el innegable despliegue del conocimiento biomédico y de su traducción tecnológica emergen apabullantes, el derecho a la protección a la salud debe manifestarse como una expresión democrática y de solidaridad.

En este sentido, el Estado mexicano debe promover efectivamente el derecho a la salud, anteponiendo no sólo el cuidado y la mejora de su salud, sino también 
su más amplio bienestar, haciendo énfasis en las poblaciones en condición de vulnerabilidad en todas las regiones del país.

Con este encuadre, el presente ensayo se propone abonar en los conceptos y delinear la participación en alcanzar la equidad en salud de la Red de Medicina de Alta Especialidad, representada por los establecimientos médicos de la Secretaría de Salud de México y agrupados en la CCINSHAE.

\section{CONCEPTOS}

La equidad en salud significa que las personas logren su máximo potencial de salud independientemente de su posición social u otros determinantes sociales.

Dichos determinantes son las circunstancias en que las personas nacen, crecen, viven, trabajan y envejecen, incluido el sistema de salud. Esas circunstancias son el resultado de la distribución de la riqueza, el poder y los recursos a nivel mundial, nacional y local, que dependen a su vez de las políticas adoptadas ${ }^{2}$.

El término equidad con frecuencia se usa con poca rigurosidad, de ahí que en ocasiones se confunda con el de igualdad, cuando en realidad son términos que expresan distintos significados. La igualdad denota propiamente uniformidad, y la equidad, imparcialidad. Por ello es de suma importancia concebir la equidad en salud dentro un concepto más amplio de justicia social.

Sin embargo, es normal hablar del tema de equidad en salud como la mera distribución de los recursos para atender la salud, cuando en realidad son muchos más los factores que intervienen en su determinación: condiciones de vida, de trabajo y ambientales, nivel de escolaridad, acceso a la cultura y participación social y política. Este es un concepto de equidad más amplio que el derivado del acceso a los servicios de salud.

Por otro lado, se entiende por inequidades en salud las desigualdades evitables entre grupos de población de un mismo país o entre países. Esas inequidades son el resultado de desigualdades en el seno de las sociedades y entre sociedades. Las condiciones socioeconómicas y sus efectos en la vida de la población determinan el riesgo de enfermar y las medidas que se adoptan para evitar que la población enferme o para tratarla. Así, existen una serie de determinantes sociales de la salud que explican la mayor parte de las inequidades en salud, esto es, de las diferencias injustas y evitables observadas en y entre los países en lo que respecta a la situación sanitaria.

Los datos probatorios indican cada vez más que los más pobres entre los pobres tienen la peor salud. Éste es un fenómeno mundial que se presenta en los países de ingresos bajos, medianos y altos. Los datos dentro de los países indican que, en general, cuanto más baja es la posición socioeconómica de una persona, peor es su salud. A esto se le conoce como gradiente social de la salud, y significa que las inequidades en salud afectan a todos. Algunos ejemplos de inequidades sanitarias entre países son:

- La esperanza de vida al nacer de las mujeres en Japón (86 años) duplica la que tienen las mujeres al nacer en Zambia (43 años).

- La tasa de mortalidad infantil es de 2 por 1,000 nacidos vivos en Islandia y de más de 120 por 1,000 nacidos vivos en Mozambique.

- El riesgo de muerte materna a lo largo de la vida durante el parto o puerperio es de sólo 1 por cada 17,400 en Suecia, pero de 1 por cada 8 en Afganistán ${ }^{3}$.

En el caso de México, la Constitución Política define la protección de la salud como un derecho, aunque ello se generó de manera paulatina, ya que en 1917 se conceptualizó la idea de protección a la salud asociada con las prestaciones de seguridad social de la clase trabajadora. En el año 1983 el concepto de previsión social en salud se adicionó al de protección de la salud en el artículo cuarto constitucional. El párrafo adicionado estipula que «toda persona tiene derecho a la protección de la salud»4.

En nuestro país no se utilizó simplemente el término derecho como en el asunto de educación, sino como derecho «a la protección», en la medida en que la salud no puede ser garantizada, sólo el acceso a los servicios médicos, así como mejorar las condiciones sanitarias y establecer medidas de prevención, todas ellas, acciones que promueven la protección a la salud $^{5}$. 
México considera que el estado de salud es un completo bienestar físico, mental y social, y no únicamente la ausencia de enfermedades, por lo que se debe comprender que fomentar la equidad en salud es vital para un desarrollo sostenible, una mejor calidad de vida y el bienestar de todos, lo cual, a su vez, puede contribuir a la paz y la seguridad ${ }^{6}$.

En este sentido, la enfermedad y la salud deben tener un lugar destacado en cualquier discusión sobre la equidad y la justicia social, no sólo por la consideración social de la salud, sino también por el carácter central que tiene la salud dentro de la justicia de los acuerdos sociales en general ${ }^{7}$.

\section{PARTICIPACIÓN DE LA RED DE MEDICINA DE ALTA ESPECIALIDAD}

Al abordar el tema de equidad relacionado con el acceso a los servicios de salud, y en particular a la medicina de alta especialidad, es importante revisar la participación que han tenido las instituciones públicas en México.

La historia de estas instituciones públicas dedicadas a la protección de la salud es un ejemplo de supervivencia y adaptaciones, condicionadas por las transiciones demográfica y epidemiológica que ha experimentado el país.

Así, con limitaciones económicas de origen y una infraestructura instalada insuficiente para atender la demanda real de la población, grupos de médicos decididos lograron el reconocimiento internacional, dando lugar a instituciones caracterizadas por las significativas aportaciones en la calidad de la atención, docencia e investigación médica.

Esta historia se remonta a las primeras décadas del siglo xx, época en la que el panorama epidemiológico se caracterizó por la presencia de enfermedades infecciosas y desnutrición como problemas prioritarios de salud pública que requerían urgentemente una respuesta social organizada 5 .

Para afrontar esta grave situación, grupos de médicos humanistas fundaron los cimientos de un sistema hospitalario con profunda orientación social, pensado para dar respuesta a las necesidades de salud de una población cada vez más grande, tendiente a la urbanización y cuya principal dolencia era la enfermedad de la pobreza.

Fue entonces que en 1943 se crearon la Secretaría de Salubridad y Asistencia -hoy en día la Secretaría de Salud-, el Instituto Mexicano del Seguro Social y el Hospital Infantil de México, considerado el primero de los institutos nacionales de salud.

Un año después, en 1944, se creó el Instituto Nacional de Cardiología; en 1946, el Hospital de Enfermedades de la Nutrición; en 1950, el Instituto Nacional de Cancerología, y en 1964, el Instituto Nacional de Neurología.

A lo largo de sus 75 años de existencia, la actual Secretaría de Salud ha experimentado transformaciones importantes en su infraestructura física, consolidación y desarrollo organizacional para cumplir satisfactoriamente con el objetivo de brindar protección a la salud de México.

Sin embargo, el panorama epidemiológico que presenta hoy en día nuestro país es muy distinto al perfil de hace unas décadas, y por lo tanto requiere de un abordaje diferente e innovador, pues los ciudadanos mexicanos ya no enferman de lo mismo, ni son las mismas circunstancias las que causan la muerte hoy en día. Por ejemplo, ya son menos los niños que mueren de manera prematura y hay menos muertes por enfermedades infecciosas.

La realidad con la que el país y su sistema de salud se enfrentan hoy en día es un aumento en el número de enfermedades crónicas degenerativas, como la diabetes, las enfermedades isquémicas de corazón, la enfermedad renal crónica y el cáncer, siendo éstas las principales causas de mortalidad ${ }^{8}$.

Esto significa que el país ha experimentado en los últimos años una transición de sus condiciones de salud, dando como resultado, por un lado, el incremento en la expectativa de vida de la población, la cual requiere servicios médicos para atender enfermedades crónicas y complejas, y por el otro, la aparición temprana de enfermedades como la diabetes y la hipertensión, resultado de estilos de vida poco saludables que prevalecen en la población y que resultan en condiciones desfavorables para la salud, como el sobrepeso y la 
obesidad, en donde México ocupa el primer lugar en cuanto a obesidad infantil a nivel mundial ${ }^{9}$.

Los retos que significan para el Sistema de Salud como resultado de esta transición demográfica/epidemiológica son muy significativos, e implican el diseño de programas y políticas públicas de promoción de la salud y prevención de la enfermedad. Sin embargo, requieren también de fortalecer la infraestructura institucional de alta especialidad, la cual es de alto costo ante el gran número de casos que requieren atención especializada.

La medicina de alta especialidad tiene como objetivo generar y profundizar el conocimiento médico por medio de investigación de vanguardia que se refleje en la implementación de nuevas estrategias y empleo de recursos tecnológicos para la prevención, el diagnóstico y el tratamiento de las enfermedades que afectan de manera prioritaria a la población.

La CCINSHAE forma parte de la estructura orgánica de la Secretaría de Salud, y como principal promotor de la medicina de alta especialidad en el país, participa de manera esencial en hacer válido el derecho a la protección a la salud que establece el artículo cuarto constitucional a través de sus entidades coordinadas.

Así, los INSALUD son los principales impulsores del conocimiento, docencia y aplicación clínica de la medicina más especializada en el país, por medio de investigación científica en el campo de la salud, lo cual los mantiene a la vanguardia para coadyuvar en atender las necesidades de salud y la formación de recursos humanos especializados, apegados a los más altos estándares de calidad técnica y con un gran sentido ético y humanístico. Sin embargo, las actividades y proyección de esta medicina de excelencia en nuestro país quedaría concentrada en la Ciudad de México, que es la sede de prácticamente todos los INSALUD, por lo que para extender los beneficios de la medicina de alta especialidad se crearon los HRAE, con el objeto de robustecer la red y extender la atención médica especializada, la formación de recursos humanos especializados y la investigación científica a diversas regiones del país. Y así, los INSALUD proyectan sus buenas prácticas en calidad y excelencia médica a las regiones y comunidades atendidas.

Esta red debe ser articulada para que las buenas prácticas de los INSALUD, por medio del personal de salud y las especialidades médicas que se ofrecen, reflejen la excelencia alcanzada por los recursos humanos formados en sus campos clínicos, con el conocimiento y, sobre todo, la mística impregnada de la investigación como cimiento de una medicina de alta especialidad.

Los HRAE deben funcionar con la excelencia que se requiere y, sobre todo, como una proyección de la experiencia de los INSALUD hacia las diversas regiones del país, cuya población merece y exige una medicina de alta especialidad.

Por lo tanto, es fundamental que los HRAE generen investigación en ciencias médicas y de la salud por medio de sus investigadores, los cuales tienen contacto directo con las enfermedades y necesidades de salud de la región, para que en un sistema vectorial de doble sentido se apoyen en los INSALUD, instrumenten y colaboren en la resolución de los problemas de salud.

Los HFR tienen como objeto principal otorgar servicios de salud en el campo de la medicina de manera complementaria y coordinada con los INSALUD y los HRAE.

En este contexto, la red integrada por los INSALUD, los HFR y los HRAE representa un esfuerzo coordinado por parte de la Secretaría de Salud para que, de manera sistemática, se lleven a cabo acciones enfocadas principalmente en lograr un acceso efectivo a servicios médicos especializados, con calidad y seguridad, en base a la mejor evidencia científica posible, la cual se vincula con los otros niveles de atención.

El objetivo es atender de manera prioritaria a poblaciones de diversos estratos socioeconómicos, poniendo énfasis en los grupos más vulnerables, contribuyendo así a disminuir las brechas que aún existen en el acceso a los servicios de atención para la salud.

De esta forma, la medicina de alta especialidad deberá generar un gran impacto sanitario y agregar valor al Sistema Nacional de Salud, al impulsar acciones e integrar procesos orientados a las necesidades de la población usuaria.

\section{CONCLUSIÓN}

En nuestro mundo globalizado existen múltiples identidades construidas en el ámbito y la influencia de la 
cultura que generan diferentes miradas acerca de la realidad. Estas expresiones culturales están relacionadas con las transformaciones históricas, el avance científico, las ideas dominantes de cada época, las percepciones religiosas, los procesos productivos y tecnológicos y las costumbres particulares de cada grupo social.

Desde una concepción amplia entendemos que el proceso salud-enfermedad está influido por múltiples factores, donde lo social tiene un peso sustancial, por lo que resulta necesario articular y recabar información de diferentes áreas; información que debemos aportar para concretar abordajes, seleccionar diversas intervenciones para situaciones diferentes y dar respuestas a quienes más las requieren.

La equidad en salud no concierne únicamente a la salud vista aisladamente, sino que debe abordarse desde el ámbito más amplio de la imparcialidad y la justicia, incluida la distribución económica y la libertad humana. No trata sólo la distribución de la salud, sino también la distribución de los servicios de salud. Es decir, debe ser entendida desde un ámbito multidimensional, cuya concepción ayuda a comprender la justicia social.

\section{BIBLIOGRAFÍA}

1. Derecho a la salud, Nota descriptiva N. ${ }^{\circ} 323$, noviembre de 2013. Disponible en: http://www.who.int/mediacentre /factsheets/fs323 /es/

2. Cisneros Luián Al. El abordaje de los determinantes sociales de la salud en México: entre el discurso y la realidad. Rev Univer Salud. 2012;15:49-56.

3. http://www.who.int/social_determinants/final_report/key_concepts/es/

4. Cámara de Diputados del Congreso de la Unión. Constitución Política de los Estados Unidos Mexicanos. Título Primero, capítulo I. De los Derechos Humanos y sus Garantías. Diario Oficial de la Federación del 27 de agosto del 2018:7-9.

5. Mayer-Serra CE. El derecho a la protección de la salud. Salud Pública de México. 2007:49:144-55.

6. OMS. Declaración política de Río sobre determinantes sociales de la salud. Río de Janeiro, Brasil, 21 de octubre de 2011. Disponible en: http://www. who.int/sdhconference/declaration/Rio political declaration Spanish.pdf

7. Sen A. ¿Por qué la equidad en salud? Rev Panam Salud Pública. 2002;11(56):302-9.

8. Lozano R, Gómez-Dantés H, Pelcastre B, Ruelas MG, Montañez JC, Campuzano JC, et al. Carga de la enfermedad en México, 1990-2010. Nuevos resultados y desafíos. Cuernavaca, México: Instituto Nacional de Salud Pública/Secretaría de Salud; 2014.

9. Villa AR, Escobedo M, Méndez-Sánchez M. Estimación y proyección de la prevalencia de obesidad en México a través de la mortalidad por enfermedades asociadas. Gac Med Mex. 2004;140:21-6. 